\title{
A new genus of lance lacewings from the Middle Jurassic of Inner Mongolia, China
}

Yiming Ma, Chungkun Shih, Dong Ren, and Yongjie Wang

Acta Palaeontologica Polonica 65 (2), 2020: 363-369 doi:https://doi.org/10.4202/app.00691.2019

Fossils of Osmylinae are rare, currently only one fossil genus of this subfamily is known, Lithosmylus which is recorded from the late Eocene Florissant Formation of USA.

Herein, we report a new Osmylinae genus with two new species, Vetosmylus tentus gen. et sp. nov. and Vetosmylus maculosus gen. et sp. nov. from the Middle Jurassic Jiulongshan Formation of Daohugou Village, Inner Mongolia, China, The new genus shares many apomorphic characters with extant osmyline relatives, e.g., forked subcostal veinlets in forewing; dense cross-veins present in radial sector, usually forming one complete outer gradate series in forewing; the region between MA and MP significantly broadened in hind wing, but with only one row of cells, implying a likely morphological stasis during the evolution of Osmylinae. The new genus Vetosmylus, the first and earliest record hitherto of Osmylinae from the Mesozoic, enhances our understanding of the early stages of their evolution.

Key words: Insecta, Neuroptera, Osmylidae, Osmylinae, Mesozoic, Asia, China.

Yiming Ma [swlsmym@126.com], College of Life Sciences, Capital Normal University, 105 Xisanhuanbeilu, Haidian District, Beijing 100048, China.

Chungkun Shih [chungkun.shih@gmail.com], College of Life Sciences, Capital Normal University, 105 Xisanhuanbeilu, Haidian District, Beijing 100048, China; and Department of Paleobiology, National Museum of Natural History, Smithsonian Institution, Washington, DC 20013-7012, USA.

Dong Ren [rendong@mail.cnu.edu.cn], and Yongjie Wang [wangyongjie@cnu.edu.cn] (corresponding author), College of Life Sciences, Capital Normal University, 105 Xisanhuanbeilu, Haidian District, Beijing 100048, China; and Academy for Multidisciplinary Studies, Capital Normal University, Beijing 100048, China. 
Attribution License (for details please see creativecommons.org), which permits unrestricted use, distribution, and reproduction in any medium, provided the original author and source are credited.

FaF Full text $(722.5 \mathrm{kB})$ 